\title{
The Subpleural Radiolucent Rim: A Sign of Alveolar Filling and Tachypnea
}

\author{
C. Joyce Lee Guy W. Soo Hoo Bruce M. Barack \\ Pulmonary and Critical Care Section and Imaging Service, VA Greater Los Angeles Healthcare System, \\ Geffen School of Medicine at UCLA, Los Angeles, Calif., USA
}

A uniform 2- to 3-mm radiolucent line adjacent to the pulsating heart or aorta in acute pulmonary edema has previously been described [1]. We observed a similar uniform 2- to 3-mm subpleural radiolucent line adjacent to the chest wall in a patient with pulmonary edema and concomitant tachypnea.

A 51-year-old man, with dialysis-dependent end-stage renal disease, was admitted with increasing respiratory distress attributed to sepsis. He developed worsening dyspnea and pulmonary edema after transfusion. An AP chest roentgenogram demonstrated pulmonary edema with radiolucent lines outlining the aorta and left heart border (white arrows), as well as right and left lungs (black arrows) (fig. 1A). These findings were also seen on a CT scan of the chest obtained after dialysis the next day (fig. 1B).

The radiolucent line outlining the heart or aorta on plain chest roentgenograms in pulmonary edema was attributed to the 'pumping effect' of the pulsating heart and aorta, which milks edema fluid from the adjacent lung, increasing lymphatic return; this re-aerates that portion of the lung, creating a 'kinetic border line' [1]. A similar mechanism likely accounts for the subpleural peripheral radiolucent rim noted in our patient. Rapid respiration and subsequent rapid chest wall and diaphragmatic movement provide a similar pumping effect that leads to the radiolucent line. We have also observed this subpleural radiolucent line with other alveolar filling processes including alveolar hemorrhage and alveolar proteinosis. This subpleural line is best seen in alveolar filling processes in which the patient is tachypneic, and may reflect the severity of the alveolar filling disease.

\section{Reference}

1 Steckel RJ: The radiolucent kinetic border line in acute pulmonary oedema and pneumonia. Clin Radiol 1974;25:391-395.

Fig. 1.
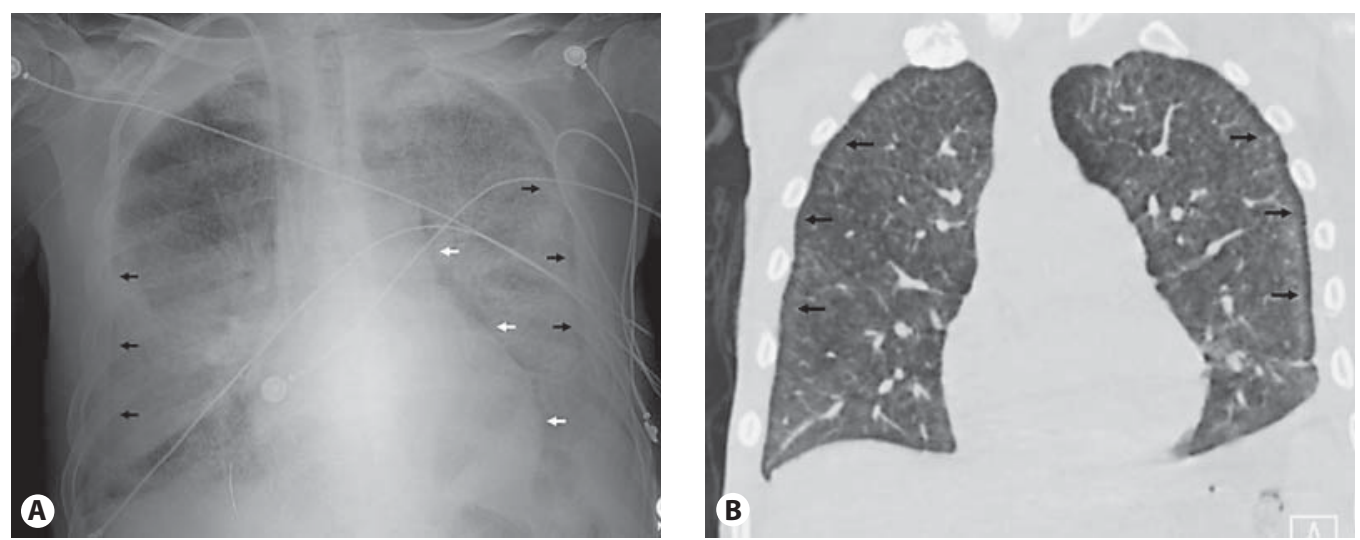

\section{KARGER}

Fax +41613061234 E-Mail karger@karger.ch www.karger.com
(C) 2010 S. Karger AG, Basel

0025-7931/10/0804-0347\$26.00/0

Accessible online at:

www.karger.com/res
Guy W. Soo Hoo, MD, MPH

Pulmonary and Critical Care Section (111Q)

West Los Angeles VA Healthcare Center

11301 Wilshire Blvd., Los Angeles, CA 90073 (USA)

E-Mail Guy.Soohoo@va.gov 